\title{
LA CONSTRUCCIÓN DE LA PERSONA EN \\ L'ACTION (III, 2-4) DE MAURICE BLONDEL
}

\section{THE BUILDING OF THE PERSON IN L'ACTION (III, 2-4) OF MAURICE BLONDEL}

John Cuadros Angulo*

\begin{abstract}
RESUMEN
Ser y actuar, o mejor, actuar para ser. Para el filósofo de la acción nos jugamos la vida en este esfuerzo por ser cada vez más nosotros mismos, y manifestarlo así en cada uno de nuestros actos. Nuestros actos manifiestan quiénes somos y pueden condicionar nuestro modo de ser o quiénes seremos. Estamos "obligados" a actuar, somos determinadamente libres, el reto será justamente el de dirigirnos, "actuando", hacia la realización de nosotros mismos, y no hacia nuestro aniquilamiento. En ese sentido, para Blondel ningún acto es insignificante, pues puede sumar a la construcción de la propia persona, o puede minar su propio desarrollo.
\end{abstract}

\section{PALABRAS CLAVE}

Acción, educación, libertad, necesidad, persona, conciencia.

\begin{abstract}
Being and acting, or rather act to be. For the philosopher of action we play life in this effort to become more ourselves, and so manifest in each of our actions. Our actions demonstrate who we are and can condition the way we are or who we will be. We are "forced" to act determinedly. We are free to act; the challenge will be just arrive "acting", towards the realization of ourselves, not to our annihilation. In that sense, for Blondel, no act is insignificant, it can add to the construction of the person, or can undermine their own development.
\end{abstract}

\section{KEY WORDS}

Action, education, freedom, need, person, consciousness.

* Universidad Femenina del Sagrado Corazón: johncuadrosa@unife.pe 
"La construcción de la persona en L'Action (III, 2-4) ${ }^{1}$ de Maurice Blondel" es el título que he querido dar a este sencillo artículo. Me he limitado aquí al estudio de una sola obra de Blondel (o mejor, a una parte de ella), y aunque sea su obra maestra, no podemos decir que agote todo su pensamiento sobre el tema. ¿Es o no importante la acción en la formación de la persona?; ¿por qué es importante?; ¿cómo se manifiesta esta importancia?; ¿qué cosa concluimos de todo esto? Son algunas de las preguntas que trato de responder con este trabajo.

Comencemos diciendo que la propuesta de Blondel se mueve en clara referencia a la línea espiritualista que caracteriza el pensamiento francés del siglo XIX, pero su espiritualismo es netamente cristiano (Urdanoz, 1988). El inicio de éste se debe a Main de Biran², para el cual sentir es actuar y en la actividad se manifiesta el espíritu. Se desenvuelve así toda la introspección biraniana, que viene a robustecer la tradición interiorística que se mueve desde Pascal, y más que a las condiciones a-priori del pensamiento mira a la experiencia de éste, al darse de la realidad en la conciencia sin todavía circunscribirse trascendentalmente el valor.

La ascendencia más directa y notoria es aquella que colega Blondel a OlléLaprune (D'Agostino, 1999), sin negar los evidentes influjos de Aristóteles, Kant, Pascal, Leibniz, Newman y Lachelier entre otros (Reale et al, 1995). Si bien es oportuno considerar la profundización de la concepción espiritualística de Ravaisson y Boutroux, se debe reconocer que subsiste un nexo entre la filosofía praticante que Ollé-Laprune proponía y la temática blondeliana del actuar; entre la indicación que sólo la práctica del bien consiente en el exacto conocimiento de las verdades morales, decisivas para el hombre, y el disponerse de estos a la acogida de la verdad cristiana por la imposición de la centralidad de la acción (Crippa, 1989).

Dividida entre el aristotelismo, que subordinaba enteramente la praxis a la teoría, y el kantismo, que ponía la praxis al primer lugar pero que era luego obligado a separarla irremediablemente de la teoría, la filosofía, para el joven Blondel, necesitaba finalmente una vía media que mantuviera el rol superior de la praxis, salvando su unidad con la teoría. (D'Agostino, 1999, p. 9). Por eso Blondel quiere integrar en modo concreto todas las dimensiones de la existencia humana y del mundo que nos rodea en una síntesis que sea comprensiva y que al mismo tiempo respete los diversos aspectos de lo real; es decir, se propone construir un realismo integral (Leclerc, 2000). En este sentido considera importante tomar en cuenta, por un lado, los datos más decisivos de las ciencias y, por otro, la apertura espontánea del hombre a un más allá de sí mismo, es decir, a una trascendencia que es propia de la inmanencia humana.

"Blondel buscaba una vía que lograra ser realista y espiritualista al mismo tiempo, que tuviera en cuenta las exigencias del ser y del pensamiento" (D'Agostino, 1999, p. 44). Este nuevo realismo, justamente porque pretende ser integral, deberá descubrir un punto

1 Para las citaciones en español de L'Action seguiré la edición realizada por Isasi e Izquierdo (1996); pero conservaré la numeración de las páginas de la edición francesa.

2 Mein de Biran polemiza contra el sensismo candillaquiano y quiere recuperar en el sensiente aquello que lo hace tal, cogiendo en la experiencia del esfuerzo la presencia de una actividad que, si bien está ligada al cuerpo, se propone como no reducible a él (Crippa, 1989). 
de partida indiscutible, críticamente justificado y un método que sea al mismo tiempo dúctil y riguroso a fin de hilvanar poco a poco todo el contenido de su pensamiento concreto; es decir, debe ser un realismo integral y también crítico, no puede ni regirse por postulados arbitrarios, ni quedarse detenido en un escepticismo deliberado (porque si no se convertiría, en ambos casos, en un dogmatismo con matices diversos). El estudio de Blondel, entonces, tratará de descubrir aquello que es universal y necesariamente implicado en nuestros actos, en nuestras palabras, en nuestros pensamientos; aquello que allí se encuentra, en primer lugar en modo escondido, implícito, y que va a continuación poco a poco (lo más completamente posible) explicitado. Es necesario, además, ser conscientes que no será nunca posible explicitar entera y adecuadamente todo lo implícito primario de nuestro conocimiento y de nuestra acción; por eso Blondel realizará una filosofía abierta, es decir, consciente de su carácter incompleto (Leclerc, 2000).

\section{La persona se manifiesta en sus actos}

Es conocido el refrán popular dime con quién andas y te diré quién eres, y en la óptica de nuestra reflexión trabajo tras los pasos de Blondel a quien podríamos parafrasear diciendo dime cómo actúas y te diré quién eres, ya que como veremos, la persona se manifiesta a sí misma a través de la acción (o mejor, de su acción). De hecho, la acción no es un accidente en la vida de la persona humana, sino todo lo contrario; más aún, la acción se presenta como "momento" privilegiado en la captación de la persona y puede servir como fuente de conocimiento de la misma, ya que "la acción revela a la persona, y miramos a la persona a través de su acción" (Wojtyła, 1982, p. 12-13).
Hemos subdividido nuestro estudio entre la presentación de la persona, su acción y su manifestación a través de la acción. Pero es evidente que esta subdivisión es solamente metodológica ya que la persona es siempre una sola en sí misma y en su actuar, o mejor dicho, es en su actuar que se manifiesta en modo evidente su unidad e identidad.

\subsection{La persona que actúa}

Queremos ver en primer lugar quién es esta persona, tal como viene presentada por nuestro autor en esta parte de L'Action. Evidentemente lo haremos de forma breve, sucinta y seguramente no exhaustiva, ya que, nuestro objetivo aquí no es el de presentar la persona en el pensamiento de Blondel, sino más bien los elementos claves que logramos captar en las tres etapas de la tercera parte que nos hemos propuesto estudiar. Dar una definición de persona es una tarea más bien imposible ya que todos los términos que utilizaríamos no serían suficientes para definir qué es, o mejor, quién es una persona. Tampoco Blondel pretende definir la persona, pero se refiere a ella de muchas maneras y con expresiones diversas, de modo que podemos captar, en los textos que estudiamos, algunos elementos claves para hacer una descripción conceptual más o menos completa de la persona.

Tal vez la primera característica de la persona que sale a la luz, es su realidad de compuesto corporal - espiritual en una realidad única. Para hacer esta afirmación nuestro autor no duda en dar razón al pueblo (o a la sabiduría popular) para quien - dice - "la persona es la unidad indistinta de una vida corporal y espiritual a la vez" (Blondel, 1983, p. 186). Y precisamente porque la persona es una unidad, es no sólo ilusoria sino sobre todo peligrosa la pretensión de formar 
dentro de uno mismo compartimentos estancos. Más aún, dirá Blondel (1893), así como "en el estudio del esfuerzo y de la resistencia orgánica no se ha podido separar la inercia material y la oposición psicológica que es su verdadera explicación, tampoco aquí, en el estudio de la acción y del papel que desempeña para la formación de la individualidad, se podría aislar la sinergia de los órganos y la armonía de las funciones psicológicas" (p. 184). La acción, entonces, se presenta con un carácter sintético (lo veremos más detalladamente en el punto siguiente): es la síntesis del querer, del conocer y del ser, que hace posible que la metafísica, la ciencia y la moral se encuentren relacionadas solidariamente (1893). Es por esto que Izquierdo (1996) considera que para Blondel el problema humano consiste sobre todo en lograr la síntesis entre realidades que una cierta filosofía (sobre todo aquella kantiana) presenta como separadas y que, sin embargo, el hombre vive y necesita vivir en unidad.

La individualidad humana aparece, entonces, como una síntesis que es a la vez orgánica y psicológica, pero es importante también aclarar que esta síntesis es el resultado de una sinergia. Esta sinergia se revela particularmente importante ya que el "resultado" del que acabamos de hablar no es ni inmediato ni definitivo; por eso, Blondel (1893) afirma que "para que la unidad del individuo se mantenga y se confirme, una cooperación constante debe aunar esfuerzos, y el equilibrio continuamente amenazado debe ser continuamente restablecido" (p. 197). Se trata por eso de un esfuerzo constante. Nuestro autor considera también el rol de la conciencia y de las facultades intelectivas de la persona, pero afirma claramente que así como la conciencia no es toda la ciencia, tampoco es toda la persona (Blondel, 1893). La conciencia resulta de una serie de actos elementales e inconscientes; no solo de uno último, sino de toda la serie que hunde sus raíces en lo orgánico y animal. Constituye una síntesis de todos ellos y un acto distinto y nuevo que, a su vez, prepara y esboza una serie de actos nuevos, cuyo término no prevé, pero que se propone como fin al menos provisorio (Hourton, 1961).

El interés de Blondel evidentemente es el de evitar cualquier tipo de visión parcial de la persona que la reduzca simplemente a una de sus manifestaciones o facultades. Con todo, no se niega el hecho de que a través de nuestras facultades logramos conocer la verdad; más todavía "... la verdad no es viviente, amante y amada más que en un espíritu vivo. Sólo llega a ser personal si proviene de una persona" (Blondel, 1893, p. 244). Como vemos, el filósofo di Aix da mucha importancia a la dimensión subjetiva en la vida concreta de la persona, lo que no significa caer en un subjetivismo. El hombre, dotado de inteligencia y de libertad siente dentro de sí la llamada a ser siempre más dueño de sí mismo y de la propia existencia; posee la capacidad de la reflexión que, aunque deriva de la espontaneidad, se libera de ella al explicarla; y aunque procede del determinismo, lo supera para conocerlo (Blondel, 1893); he aquí una vez más expresa la grandeza del hombre.

De libertad hablaremos con más detalle en el punto siguiente, por ahora sólo podemos decir que ella se presenta también como una dimensión esencial de la persona, ya que en ella vemos aparecer un impulso y una necesidad a salir de sí misma que es fundamental también en el individuo para que surja la persona. En efecto, "lo mismo que la libertad formal no ha salvado su autonomía más que al imponerse la heteronomía de una obligación práctica y de un esfuerzo, de igual modo, la persona no nace en el individuo, no se constituye 
y conserva más que al asignarse un fin impersonal" (Blondel, 1893, p. 198). Es evidente, entonces que el hombre no se basta a sí mismo; no sólo necesita actuar con los demás, sino también para los demás y por los demás, ya que no puede solucionar por sí mismo todos los asuntos de la propia vida.

Por otro lado, no podemos negar el hecho de ser libres. La libertad tiene que ser aceptada, ya que no depende de nosotros el ser o no ser, a nuestros propios ojos, libres (Blondel, 1893). De hecho hasta el mismo determinismo, del cual hablaremos más adelante, está subordinado a una libertad más íntima. He aquí la importancia de ser cada vez más concientes de las propias capacidades, sobretodo en el ejercicio de ellas. Podríamos decir que se trata de ser concientes de la propia libertad y libres en la propia conciencia ya que "la reflexión y la libertad son imposibles en quien, en lugar de actuar fuera actuado. Porque no hay razón ni conciencia refleja, ni sentimiento de infinito más que donde hay actividad libre, y no hay actividad libre más que allí donde hay conciencia de actuar" (Blondel, 1893, p. 120).

Aparece aquí la importancia y la necesidad de la dimensión "impersonal" del individuo ya que "sin lo impersonal percibido vagamente, no nos veríamos como en un cristal transparente. El egoísmo nos ciega. Si salimos de la vida individual, si nos adherimos a algo distinto de nosotros, entonces nos poseeremos mejor" (Blondel, 1893, p. 199). Esta es una verdad muy grande ya que "nuestra vida es el concurso de todo lo demás; nuestra persona es nuestra expansión y nuestra dedicación a todos; nuestra acción es la colaboración del universo y el triunfo de la impersonalidad" (Blondel, 1893, p. 200). Es evidente que hablamos aquí de una impersonalidad que no es ni destructiva ni alienante para la persona, sino más bien la puerta hacia el conocimiento de su misma interioridad y hacia la trascendencia de sí mismo en el contacto y en la vida con los demás. Podemos concluir, junto a Blondel (1893), que en síntesis "la sustancia del hombre es la acción, él es lo que hace [...] Nosotros no somos, no conocemos, no vivimos más que sub specie actionis" (p. 197). Por eso, nos dedicaremos un poco a ver la persona en su acción o, en otras palabras, el acto de la persona.

\subsection{El acto de la persona}

Definir la acción no será nuestra tarea. Siguiendo a nuestro autor queremos presentar la acción de la persona, pero no pretendemos dar una definición de la acción. De hecho, el mismo Blondel no sólo no ha dado una definición, sino que "incluso ha considerado imposible esta tarea, dado que la acción no es la idea de la acción, y la realidad de la acción sólo puede ser captada en su realidad con la práctica" (Izquierdo, 1996, p. xxviii). Nuestro objetivo, no lo olvidemos, es sobre todo ir descubriendo poco a poco cómo la realidad de la acción no sólo manifiesta la persona, sino que también la construye.

\subsubsection{La acción como síntesis...}

El apartado que tratamos ahora lo hemos llamado "el acto de la persona",

3 Nos limitamos ahora sólo a presentar la distinción que Blondel hace entre estas dos dimensiones íntimamente ligadas por el hecho de ser facetas de una misma realidad: "El acto es más bien la primera iniciativa del esfuerzo interno, [...] La palabra acción indica más bien el paso de la intención a la ejecución que la encarna, [...] Entre el acto y la acción subsiste, pues, una diferencia análoga, pero contraria, a la que se da entre obra y operación" (Blondel, 1893, p. 116). 
pero no haremos mayor distinción entre el acto y la acción de la persona, visto que éste no es el objetivo de nuestro estudio $^{3}$. Lo que sí debemos tener siempre presente es que en Blondel el término Acción «denota todo el encadenamiento de la actividad humana, desde su sustrato cósmico y orgánico hasta la síntesis superior del pensar y del queren» (Hourton, 1971, p. 327); no somos sólo el producto de un ambiente, sino causa o concausa de él (Hourton, 1961). De este modo, el término acción para Blondel comprende toda actividad específicamente humana, metafísica, moral, estética, científica, práctica (Baravalle, 1972).

Al actuar, el individuo sale de sí mismo, su vida no puede limitarse a sí misma. El hombre, si realmente piensa y reflexiona sobre su existencia, descubre siempre en él insuficiencia, penuria y falta de equilibrio entre lo que realmente quiere y lo que finalmente logra (Hourton, 1961). De hecho, "sólo va hacia adelante el que no tiene, ni detrás de sí ni tampoco en el lugar en el que está, seguridad o suficiencia" (Blondel, 1893, p. 198); es por eso que hasta en el querer aparentemente más insignificante se encuentra otro, y en éste está contenido otro más, y así sucesivamente hasta que se llegue a un querer que satisfaga definitivamente el espíritu humano. "El hombre [...] no actúa, pues, según la idea que tiene de la acción, sino en la medida en que es principio y fin de sus actos" (Blondel, 1893, p. 126).

En su actuar, el hombre se ve implicado alma y cuerpo, y la acción se presenta como el nexo de la síntesis orgánica y de la individualidad consciente. El más pequeño acto consciente y querido mete en acto todo su ser, de modo que la acción es una prolongación del propio querer fuera de uno mismo (Blondel, 1893). La acción, entonces, se presenta como parte integrante de la intención, a la que vivifica e ilumina. Así, sobretodo la operación motriz, pero no sólo, supera inevitablemente las fronteras del individuo, evidenciando el rol del cuerpo como intermediario de la misma libertad en este diálogo de interacción entre el hombre y el ambiente que lo rodea. En conclusión, "la acción es una concentración sistemática de la vida difundida en nosotros; es una toma de posesión de sí mismo" (Blondel, 1893, p. 194). Cada acto nuestro, en cuanto concentra en sí todos los pensamientos y sentimientos para conferirles el sigilo de una decisión ideal, es acto metafísico. La historia de un hombre es una metafísica viviente (Grillo, 1970).

Pero es claro que actuar no es sólo actuar físicamente, ya que el pensamiento es también una forma de acción y concebir significa haber actuado, seguir actuando todavía y deber actuar más allá (Blondel, 1893); "sólo en la conciencia hay que buscar ahora el principio interno, el principio determinante de la acción" (Blondel, 1893, p. 104). En todo caso, la primacía de la acción es clara, ya que "no se piensa (éste es el orden) más que después de haber actuado, y al actuar, y para actuar" (Blondel, 1893, p. 109). Así con el término acción se designa algo de "anterior" (y por eso en cierto sentido superior) al entendimiento reflejo y al pensamiento discursivo, pero se designa también algo de coincidente y concomitante, y finalmente también algo de posterior y consecuente con la acción (Vedaldi, 1950). Aunque a simple vista no se perciba, en una acción hay algo más que la acción; se encuentran también la cohesión, la solidaridad, la unión real de todo lo que emplea y de todo lo que con ella colabora. Así, para Blondel (1893) "todo acto es en su origen una unidad indivisible en la que se encuentran la iniciativa humana y la contribución del universo" (p. 196), porque "nuestra acción 
es la colaboración del universo y el triunfo de la impersonalidad" (p. 200). Pero el triunfo de la impersonalidad no significa que se trate de un actuar irracional, al contrario, ya que el hombre tiene la capacidad de comprender todo sistema de ideas complementarias y a la vez paralizar su influencia inmediata para reflexionar y deliberar sobre ellas. A esto comúnmente se le llama la razón. De este trabajo interior surgirá el acto humano, el acto razonable, el acto voluntario, el acto libre, o simplemente el acto o la acción (Blondel, 1893). Un acto, entonces, que no es razonable y razonado, no se podrá llamar ni libre ni voluntario. El auténtico actuar del hombre será entonces aquél que, siendo razonable, es libre y voluntario. "Los fenómenos, aunque todavía ajenos a la vida interior, reciben su alma y su conciencia en la voluntad que los emplea en la acción" (Blondel, 1893, p. 196).

\subsection{2 ... entre libertad...}

Entra así, en nuestro discurso, el tema de la libertad porque uno no puede ser consciente de una acción cualquiera, sin sentirse al mismo tiempo libre (Arrieta, 1993). "Para actuar hay que participar de una capacidad infinita. Para tener conciencia de actuar es necesario tener la idea de este poder infinito. Pero es en el acto racional donde hay síntesis de la capacidad y de la idea infinita. Y a esta síntesis es a lo que llamamos libertad" (Blondel, 1893, p. 121). Se trata de un poder infinito que al mismo tiempo, contrario a lo que muchas veces se piensa, no es un simple poder arbitral, capaz de prestar o de rehusar la mediación de la razón. "Ella ha salido del dinamismo de la acción espontánea, y por eso tiende necesariamente al dinamismo de la acción refleja" (Blondel, 1893, p. 125).

Por otro lado, la libertad es una dimensión dinámica del hombre porque ni se conserva ni se desarrolla sino superándose a sí misma. Se trata de una aparente abdicación, dice Blondel (1893). Pero, ¿uno puede escoger no ser libre? La respuesta es simplemente no, ya que "la libertad, necesariamente producida en la conciencia, se ejerce también necesariamente" (p. 124), y decidir no ser libre es ya un acto de libertad. La libertad germina, crece y fructifica necesariamente. Ella no escapa a la necesidad de ser y de mantenerse como la razón de la acción. "La libertad, que se produce fatalmente, no se conserva sino libremente" (p. 129), es decir, por una fidelidad a la lógica misma de la libertad que es querer infinitamente (Hourton, 1961). La misma muerte, al "destruir" la libertad de actuar, no hace más que consagrar la libertad de no actuar (Blondel, 1893). No se puede, entonces, no ser libres, y la libertad no se diferencia del uso que se hace de ella. "Si abdica y se somete, es inmanente a su propia decadencia; si se libera y se desarrolla, es inmanente a su propia trascendencia. De todas formas, la voluntad refleja es siempre doble, porque es a la vez un principio de acción y un fin a alcanzar" (p. 133). Se trata, entonces, de descubrir el deber en la libertad misma y de encontrar, en lo que ella no es todavía, el deseo secreto de lo que ya es.

Ser libres, por eso, es un camino de toda la vida, un camino nunca terminado, un desafío que está siempre frente a nosotros, ya que nadie está obligado a seguir siendo libre, aunque el negarse a ser libre es ya un acto de libertad, y "la libertad sólo se hace real al querer serlo. Siendo necesariamente producida, sólo es ella misma al reproducirse" (Blondel, 1893, p. 132). Así, "la libertad libre es aquella que quiere querer, aquella que, al suprimir en primer lugar la eficacia natural de las tendencias espontáneas, consiente en sufrir la atracción de una de ellas sólo en la medida en que ella pone la razón de 
su decisión" (p. 131). Por este motivo "en relación con la conciencia, la razón del acto no puede residir más que en una libertad capaz de sintetizar, de sacar provecho y de superar todo el determinismo del que ella ha salido y acepta" (p. 127); esto se explica porque lo mismo que la libertad no se halla separada de sus antecedentes necesarios, tampoco está separada de sus necesarias consecuencias. Es un camino que debe recorrer la libertad humana, o mejor, el hombre con su libertad. Así, de una libertad que al comienzo no era más que una exención de la necesidad antecedente (immunitas a necessitate), se pasa a "una voluntad plena y dueña se sí misma, una voluntad que sabe y puede querer, una voluntad liberada poco a poco de lo que le impide ver, querer y hacer, una libertad más libre, immunitas a servitute, liberum consilium" (p. 196). Y ¿cuándo es que hemos tomado conciencia de nuestra libertad? Al actuar. Y es necesario que actuemos para desarrollar nuestra libertad y conocerla mejor (Blondel, 1893). Se trata de un tesoro que no debe ser "conservado" celosamente sino desarrollado con esfuerzo y generosidad. El designio más profundo de la voluntad es la búsqueda de un acrecentamiento, de un acabamiento, de una acción que iguale al querer (Hourton, 1961).

\subsection{3 $\ldots y$ y determinismo...}

Junto al discurso sobre la libertad surge aquél sobre el determinismo, y paradójicamente "no hay conciencia del determinismo más que por la libertad. La libertad adopta todas sus condiciones antecedentes. Pero no encuentra en ellas su razón de ser. La razón verdadera de la acción se debe descubrir en un fin trascendente a la naturaleza o a la ciencia" (Blondel, 1893, p. 124). Por eso no es cierto que al afirmar la verdad del determinismo se esté negando la libertad; se trata, sí, de dos diversas formas de fenómenos, pero eso no quiere decir que sean incompatibles. Al contrario, por una parte, el determinismo ha desembocado necesariamente en la conciencia de la libertad; por otra, la libertad, al tomar conciencia de sí misma, ratifica todo lo que la precede y quiere todo lo que le permite querer. "La libertad, lejos de excluir el determinismo, brota de él y de él se sirve. El determinismo, lejos de excluir la libertad, la prepara y la produce" (p. 120). En este sentido es clara una triple verdad:

1) nada en el determinismo de la naturaleza y del pensamiento, nada en los fenómenos ni en la ciencia de los fenómenos contradice la conciencia de una fuerza exenta de necesidad, immunitas a necessítate; 2) son el movimiento de la espontaneidad y el desarrollo del determinismo los que producen la conciencia necesaria e inevitable de la libertad, necessitas libertatis; 3) el juego mismo de este determinismo es explicado y consagrado por la libertad, necessitas a libertate (Blondel, 1893 p. 121).

Pero como la libertad es un camino a seguir en el crecimiento personal y el hombre es una unidad corporal-espiritual, no debemos olvidar que "así como la verdadera iniciativa de la voluntad supera el automatismo inconsciente y las condiciones fisiológicas del acto, del mismo modo la resistencia orgánica, lejos de limitarse a la inercia bruta, afecta sobre todo al despertar en nosotros de estados de conciencia extraños u hostiles a la conciencia, de nuevas voluntades que se alzan contra la voluntad"(Blondel, 1893, p. 166). Es la constatación que todos más o menos hacemos: "quiero actuar, y actúo más o menos. Quiero querer, pero en el instante de ponerme a ejecutar y de pasar al acto no quiero" (Blondel, 1893, 
p. 166). Aparece claro que la acción sea una conquista, hablamos evidentemente de la verdadera acción, aquella que se puede llamar tal en pleno sentido porque consciente y libre. "La acción es la intención que vive en el organismo y modela las energías oscuras de las que había emergido. En efecto, es necesario que la libertad se despliegue y se encarne para conservarse y desarrollarse" (p. 144); ya que "cuanto menos capaz se es de actuar, más desenfrenada se hace la intemperancia de los deseos, porque el equilibrio entre los sueños y la práctica cotidiana se rompe cada vez más" (p. 184).

\subsection{4 ... para un actuar más consciente}

La libertad necesariamente producida y necesariamente ejercida en nosotros sólo se mantiene libre bajo la forma de una determinación, de una obligación y de una acción. "De este modo, para que la voluntad siga siendo sincera, se debe pasar de la autonomía a la heteronomía, y del formalismo de la intención a la producción de la acción, y así es como supera el alcance de la conciencia" (Blondel, 1893, p. 128). Es evidente, entonces, que la heteronomía no es contraria al deseo profundo de la libertad y que la ley moral resulta indispensable para la libertad. Incluso cuando la voluntad libre se considera a sí misma como un fin, lo que quiere es algo distinta de ella. Y es que, "al proponerse la libertad como fin, se siente una desproporción entre la voluntad que quiere, quod procedit ex voluntate, y la voluntad querida, quod voluntatis objectum fit" (p. 132) ${ }^{4}$; se trata de una inadecuación, de una diferencia tal que no permite paradas definitivas
(Baravalle, 1972). De esta forma aparece claro que "la voluntad deviene, se hace; no existe nunca sin cambiar y no se conserva sino dándose" (Blondel, 1893, p. 141). "Nacida así de la acción, mantenida por la acción, perfeccionada por la acción, la conciencia de la libertad y de la obligación moral tiende a la acción" (Blondel, 1893, p. 139). Por eso la acción es siempre un más allá, y en adelante "es de la acción de donde se debe partir como el verdadero y sólido origen del movimiento que lleva al hombre hacia fines conocidos y queridos" (p. 122), o podríamos decir, mejor, que es a través de la acción como podemos constatar lo que realmente se quiere y se desea. La intención moral necesita de la acción y a través de ella se manifiesta, ya que "es por la acción, en efecto, como la intención moral se insinúa en nuestros miembros, hace latir nuestro corazón y fluir su vida propia en nuestras venas; es por la acción como vuelve a la conciencia, más llena, más clara y cargada con sus conquistas" (p. 139). El hombre descubre, entonces, ante sí un deber que es necesario reconocer, es decir, tomar conciencia de él y de lo que pide, ya que este deber

impone a la libertad íntima no solamente el respeto y la sumisión interior, sino un esfuerzo y unos sacrificios reales, un crédito y una generosidad cuyo origen no está en el mero conocimiento que tenemos de la ley, un acto positivo que supera esencialmente la conformidad puramente formal de la intención con el deber (p. 134)

Aparece entonces evidente cómo la libertad misma no es un motivo

4 La distinción que hace Blondel entre "voluntad que quiere" y "voluntad querida" no es objeto de nuestra reflexión, por eso sólo la mencionamos de forma breve. Sobre el tema de la voluntad en L'Action, se pueden revisar las reflexiones de Virgoulay (1987) y Marion (1990) al respecto.

5 El formalismo al que se refiere Blondel en este párrfo no puede ser otro que aquel de Kant. 
meramente formal, ya que ella "se encarna necesariamente en los motivos particulares, y cuando se distingue de ellos no los suprime" (Blondel, 1893, p. 130). Al contrario, porque si es verdad que al principio es necesariamente inmanente a un motivo cualquiera entre otros motivos igualmente determinados, al final la libertad se distingue de todos ellos y da al objeto preferido un carácter de trascendencia, como trascendente es la libertad misma. Finalmente "no hay razón, ni conciencia refleja, ni sentimiento de infinito más que donde hay actividad libre, y no hay actividad libre más que allí donde hay conciencia de actuar" (Blondel, 1893, p. 120); por eso, a mayor conciencia, mayor libertad. Pero en conclusión ¿qué significa actuar, - se pregunta Blondel (1893) - según la idea común que nos hacemos de ello? "Significa introducir y añadir algo de uno mismo en la inmensidad de las cosas que envuelven siempre y en todo un infinito actual, en el ámbito del determinismo que afecta a toda la complejidad de los fenómenos" (p. 118); en otras palabras, ir más allá de uno mismo y dejar una huella de nuestra existencia, porque a fin de cuentas esa acción será nuestra y sólo nuestra.

\subsection{La acción como manifestación de la persona}

"Sólo subsistimos en la medida que actuamos" (Blondel, 1893, p. 182). Parecería una afirmación demasiado aventurada $\mathrm{y}$, sin embargo, es evidente que sólo al actuar y actuando es como hacemos presente nuestra existencia y subsistencia. Se da un progreso de la acción en el ser y un progreso del ser por la acción, y esto no es indiferente, porque el que actúa es siempre el ser humano, y es en su ser que puede actuar y es a través de su acción que puede ser siempre más. Blondel tiene una expresión clara y precisa que no deja dudas sobre el valor que él daba a la acción como manifestación de la persona y como "instrumento" para su crecimiento: "No solamente la acción manifiesta lo que ya éramos, sino que también nos hace crecer y, por así decirlo, salir de nosotros mismos" (1983, p. 197). Este salir de nosotros mismos implica evidentemente que la acción nos lleva continuamente fuera de nuestra individualidad, sin negarla ni destruirla; al contrario, justamente para construirla y hacerla más auténtica. Lo queramos o no, seamos o no conscientes de ello, con nuestra presencia y con nuestra acción nos propagamos alrededor de nosotros mismos. La acción "sacude y pone en movimiento toda la máquina [todo el hombre]. Desde el momento en que la operación querida se realiza en nosotros, hay de hecho sinergia y concurso" (Blondel, 1893, p. 187). Lo hemos visto ya en el punto anterior y por eso sólo lo recordamos, porque es importante no perder de vista la unidad integral del ser humano. Somos una unidad aún en los momentos en los que terminamos haciendo lo que no queríamos hacer o nos habíamos propuesto no hacer; o bien no hacemos lo que queríamos y nos habíamos propuesto hacer. Es verdad, "no hemos querido todo lo que hacemos y, bajo el imperio de la pasión terminamos queriendo lo que no queríamos. ¿Qué significa? Que la acción nos revela lo que queremos y por ende, lo que somos" (Hourton, 1961, p. 63).

Para comunicar nuestras experiencias, lo que hacemos o hemos hecho y vivido, hacemos uso de la palabra o de palabras. Se trata de una característica propia del ser humano: la capacidad de comunicar verbalmente. Pero "si toda acción puede ser expresada y explicada por la palabra, ello significa, en primer lugar, que toda acción es una palabra implícita, es decir, una necesidad de revelarse a todos" (Blondel, 1893, p. 232). "Un gesto 
vale más que mil palabras", dice un conocido refrán, y es verdad: ¿no es con nuestros actos o modos de actuar que expresamos alegría, dolor, rabia? Pero no sólo sentimientos y estados de ánimo, sino también nuestros valores, creencias, etc. "La acción no sirve solamente para revelar lo que en nosotros es más fuerte, o incluso a veces lo que es más fuerte que nosotros, sino que también constituye a menudo, en medio de la indiferencia y del desarraigo de los estados interiores, un centro sólido que se convierte como en el núcleo del carácter" (p. 189). De hecho, ¿no nos ha sucedido alguna vez que hemos actuado en un modo que ni los otros ni nosotros mismos nos hubiéramos esperado? La acción se convierte de esta forma en un espejo que nos permite ver la imagen visible de nuestro carácter. Surge así un desafío evidente pero que no siempre se puede dar por descontado y es el de no menospreciar el más mínimo acto que realizamos. Hasta el más pequeño acto, y aparentemente insignificante o indiferente, tiene un peso y un valor. Por eso, Blondel invita a regular diligentemente el sentido (así lo llama) "del detalle ordinario, el del curso cotidiano de la vida; porque en las circunstancias decisivas somos lo que hemos sido, y entonces cambiamos en billetes grandes las pequeñas monedas que hay que juntar una a una" (p. 196).

\section{La persona se construye en sus actos}

Hemos tratado de presentar en modo sencillo la persona que actúa, el acto de la persona y la acción como manifestación de la persona. Se trata de una distinción difícil de hacer y realmente inconcebible, pero que hemos realizado sólo metodológicamente. A este punto nuestra tarea también será difícil porque hablaremos de la importancia, de la necesidad y del valor de la acción, es decir, hablaremos de la acción misma. Nuestro interés será el de descubrir, junto a Blondel, el rol de la acción en la formación de la persona.

\subsection{Importancia de la acción}

Si es verdad que se da un progreso de la acción en el ser, es verdad también que se da un progreso del ser por la acción (Blondel, 1893). Creo que no sería exagerado afirmar que se actúa porque se es y se es siempre más (o siempre menos) gracias a la propia acción. La acción juega, pues, un papel esencial en la constitución de la razón y en la idea de libertad, pero muchas veces no se la valora como se debiera. "¿Acaso la acción no será finalmente, por su propia virtud, mediadora y unitiva, redentora y perfeccionadora?" (p. 148) Se pregunta Blondel (1893), y parece como si se respondiera más adelante: "El acto tiene una eficacia suprema para obtener la unanimidad interior" (p. 189) y es que "de un modo totalmente natural la acción contiene e implica las tendencias más opuestas" (pp. 182 183). La práctica logra lo que las especulaciones abstractas no logran, es decir, unir "en una nueva síntesis tendencias opuestas que, victoriosas o vencidas, están todas ellas representadas, reformadas, transformadas en el acto realizado" (p. 193). La acción, entonces, une las fuerzas dispersas de la vida y constituye la síntesis orgánica, sirviendo de mediadora entre todas las formas de la actividad corporal y espiritual. Ella enriquece la voluntad al responder al primer movimiento de su pasión hacia el exterior con un movimiento hacia el interior, cuyo retorno compone el ritmo vital; de este modo cierra el circuito de la vida individual. La acción se convierte así en la conciliación viva de los contrarios, en el nexo de la síntesis orgánica y de la individualidad consciente, y además, forma el carácter (Blondel, 1893). 
Resulta entonces, verdad que el alma toma cuerpo por la acción y por ella también el cuerpo toma alma (Grillo, 1970). La acción, pues, es su vínculo substancial, y con ellos forma un todo natural. En este sentido "no existe un límite fácil de precisar de la cooperación del cuerpo, de su fuerza de resistencia, a su potencia moral, porque la acción lo une y lo eleva a la inagotable fecundidad de la razón y de la libertad" (Blondel, 1893, p. 186). La misma intención necesita realizarse por la acción. No hay otra posibilidad si se quiere ser coherente y no quedarse en la mera intención. En este sentido la acción enriquece la intención. La acción somete la voluntad a la estrecha sencillez de una única salida, es verdad, pero es así como aquella se convierte para ésta en vía de expansión y de enriquecimiento.

\subsubsection{Necesidad de la acción}

"Sólo subsistimos en la medida en que actuamos" (Blondel, 1893, p. 182). Es necesaria entonces la acción para garantizar nuestra subsistencia, más aún, para hacerla mejor y más auténtica. Pero no sólo eso, sino que es también actuando como tomamos conciencia de nuestra libertad, y es actuando como la desarrollamos y conocemos cada vez más: la libertad necesita desplegarse y encarnarse para conservarse y desarrollarse verdaderamente, y esto lo hará en la acción. Dice nuestro autor, "reparamos y aumentamos nuestra energía cuando usamos de ella, cuando parece que la sacrificamos y mortificamos. En el dominio de la acción voluntaria, cuanto más se gasta, más se posee" (p. 186). Nuestras capacidades, entonces, tenemos que desarrollarlas, porque no ejercitarlas, no hacerlas crecer, no es conservarlas sino limitarlas o incluso anularlas.

Cuando concebimos un acto, necesariamente están presentes, al menos como posibilidad, una serie de actos diferentes entre los cuales nos hemos decidido por uno preciso, o en base a los cuales hemos escogido el que nos parecía mejor, tal vez hasta mejorado con variaciones presentes en las otras posibilidades. En este sentido el acto por el que nos hemos decidido no sería tal en ausencia de los otros posibles. Del mismo modo, la voluntad libre está necesariamente involucrada en nuestros actos reflejos y ratifica todas las consecuencias de su intervención o de su abstención. Entonces, no se trata sólo de una posibilidad necesaria, sino que es un hecho necesario (Blondel, 1893). Pero "la acción no es acción más que en la medida en que constituye un organismo y hace concurrir a un fin único una diversidad de fenómenos" (Blondel, 1893, p. 211). Se convierte de este modo en síntesis de la persona, de sus capacidades y dimensiones, como hemos visto ya líneas arriba. Pero no sólo esto sino que también "la acción sirve de garantía de las promesas, y es como la sustancia de compromisos irrevocables, porque manifiesta, fija, confirma y hasta produce la voluntad" (p. 189). En la acción, entonces, la persona manifiesta lo que realmente quiere, tal vez, hasta de modo inconsciente. También es a través de la acción que la persona puede hacerse virtuosa (o viciosa), en comportamientos, actitudes, modos de pensar, etc. Finalmente, es verdad que muchas veces hacemos lo que queremos, pero, también es verdad que a fin de cuentas terminamos queriendo lo que hacemos. "Así, pues, se resumen todas las inconsecuencias de la acción: casi nunca hacemos todo lo que queremos; a menudo hacemos lo que no queremos; y acabamos por querer lo que no queríamos" (Blondel, 1893, p. 178). Por esto, ser verdaderamente dueños de nuestros propios actos es un desafío constante en este perenne dinamismo de nuestro actuar. 


\subsection{Valor de la acción}

"La individualidad humana es una síntesis a la vez orgánica y psicológica, y esta síntesis es el resultado de una sinergia. Así es como la vida individual se determina, el carácter se delinea, la persona recibe su forma sustancial, ya que, a veces, basta con un solo acto para transformarla" (Blondel, 1893, p. 196). El valor y la importancia de nuestros actos no residen tanto, entonces, en el hecho de que sean ordinarios o extraordinarios, grandes o pequeños, porque cada acto, hasta el más pequeño y aparentemente indiferente tiene un valor y un peso en nuestra historia y en nuestra vida. Y como somos conscientes que no siempre es fácil mantener en equilibrio la voluntad, "hay una necesidad continua de que el equilibrio perpetuamente inestable de la voluntad vaya a parar enteramente de un lado, y que, formando un único sistema con los vencedores y los vencidos, esta sinergia construya la persona" (pp. 179. 180). Es la acción la que, logrando la sinergia en la persona y la unidad en la variedad de motivos, movientes, posibles acciones, etc., la va construyendo poco a poco. Pero esto se da solo si las acciones son buenas, es decir, de acuerdo a su identidad de ser corporal-racionalespiritual, ya que de lo contrario también el hombre se puede destruir con sus acciones. En ese sentido, "la mejor higiene no consiste en cuidar el cuerpo sólo por medio del cuerpo. En el mismo ascetismo se encuentra un principio de rejuvenecimiento, de salud y de vigor" (p. 186), porque cuerpo y espíritu tienen que ser cuidados del mismo modo y, mejor incluso, sincrónicamente.

Es la acción, nuestra acción, la expresión de nuestro ser, y "no solamente la acción manifiesta lo que ya éramos, sino que también nos hace crecer y, por así decirlo, salir de nosotros mismos"
(Blondel, 1893, p. 197). Se trata de un dinámico ser actuando, y para actuar, y finalmente para ser (crecer) cada vez más. De este modo la acción se presenta como el material con el cual somos modelados. Esto es así, ya que la expresión del acto, por imperceptible que sea en el origen, es el germen de un inmenso crecimiento. Entendemos, entonces, por qué el hombre "no actúa, pues, según la idea que tiene de la acción, sino en la medida en que es principio y fin de sus actos" (p. 126), ya que es él quien actúa y genera su acción, y es siempre él el primer afectado y destinatario, si podemos decir así, de su actuar, aunque no sólo el único. Se trata de un esfuerzo que debe hacer el individuo desde su niñez. De hecho, podemos constatar que la razón ha aparecido en él cuando ha aprendido a reconocer que los otros son también un yo, distinto de sí y contra sí. Es entonces cuando él se hace una persona, y una persona entre tantas otras personas, cada una con un valor intrínseco, como el propio. Al mismo tiempo tendrá que llegar a participar, aunque sea a sus expensas, en la persona de otro, y hacer un esfuerzo sobre sí mismo para no querer ser ingenuamente el centro de todo. Sale a la luz aquí la realidad de la acción como una función social por excelencia (Blondel, 1893). Evidentemente, este es un camino que un niño no puede hacer solo. En él están presentes diversidad de deseos opuestos y de caprichos. Así como construye, destruye; se cansa rápida y fácilmente de todo, porque es "una anarquía viviente". Es necesario que logre organizar en él un sistema, que aprenda a agrupar sus fuerzas y a controlarlas. Es necesario que aprenda a seguir decididamente una de sus tendencias, excluyendo las demás, que en la anarquía en la que vive - que por otro lado es normal - son muchas y muy diversas. Será la educación la que le ayude en esta difícil pero necesaria, más aún, vital cristalización. La tentación más 
grande de padres y hasta de educadores es la de ceder ante él siempre, no molestarle y no contrariarle en nada; gravísimo error, ya que esto equivale a destruirlo sistemáticamente, porque entonces no sabrá ni comprender sus propios deseos, víctima como es de la anarquía de caprichos que viven dentro de él. El resultado será que ni él mismo sabrá lo que realmente quiere, puesto que no ha aprendido a decidirse nunca por algo en modo concreto y serio. Sus deseos no serán ya verdaderos deseos y sus actos dejarán de ser verdaderamente actos (Blondel, 1893).

Aparece claro entonces el papel que juega la acción, nuestra acción, cada acción en nuestra vida: ella no sólo manifiesta lo que somos realmente, sino que también nos construye (o destruye). Se trata de un desafío puesto ante nosotros: 1) ser cada vez más conscientes de nuestros actos, es decir, cada vez más libres; o vivir siempre sometidos a nuestros caprichos y pasiones irracionales, incapaces de decidirnos verdaderamente por algo; 2) aprender de nuestras acciones a conocernos a nosotros mismos; o ser indiferentes ante ellas y vivir, o mejor, funcionar como autómatas; 3) y, finalmente, crecer cada vez más, construyéndonos, porque libres y conscientes de nuestros actos; o destruirnos a nosotros mismos porque incapaces de autodominio y autodecisión. No nos queda otra que decidir y actuar, decidir actuando y actuar para decidir.

\section{A modo de conclusión}

Hemos descubierto la acción como realidad concreta e ineludible en nuestra existencia. No podemos no actuar. Hasta las realidades más espirituales de nuestra persona, como el querer o el pensar son actos, y en este sentido no es raro hablar de actos intelectivos o actos de voluntad. Pero no solo eso, sino que además hemos aprendido que a través de nuestros actos no sólo podemos conocernos a nosotros mismos más y mejor, sino también, podemos formarnos, crecer, o "construirnos", como hemos expresado en el título de nuestro artículo. Es clara, entonces, la importancia de detenernos a reflexionar sobre nuestros actos para descubrir en ellos nuestras más profundas intenciones y conocernos a nosotros mismos. En este sentido da pena ver como muchos, en esta sociedad moderna, no tengan tiempo para sí mismos, para pensar en lo que hacen, para hacer mejor lo que hacen y descubrirle el sentido. El ritmo frenético de la vida de muchos los lleva no a actuar - porque actuar, en el más profundo de los sentidos, es vivir - sino a funcionar; no se pesa el valor de los propios actos, no se busca el sentido, se quiere eficiencia y eficacia, cosa que podríamos exigir igualmente a máquinas inventadas justamente para ser eficientes y eficaces, aunque no sean conscientes de ello y no descubran el sentido de lo que hacen, porque no viven, funcionan. Ser conscientes de lo que hacemos y por ende más libres, para construirnos en nuestro actuar del mejor modo, es entonces, el reto que se abre ante nosotros. No hay otra: o actuamos o terminamos por ser actuados, es decir, por sufrir inconscientemente, y a veces también irresponsablemente, el influjo externo. Finalmente, no podemos correr el riesgo de perdernos o destruirnos con decisiones y acciones erradas; así como el actuar nos construye, también nos puede destruir si no corresponde a nuestro ser y deber ser. "Lograr la unidad en la totalidad de nuestro ser, y ser cada vez más dueños de nuestra propia vida", éste es el desafío que queda ante nosotros, ¿̇lo aceptamos? 


\section{REFERENCIAS}

Blondel, M. (1996). La Acción. Ensayo de una crítica de la vida y de una ciencia de la práctica. Madrid: BAC.

Blondel, M. (1893). L'Action. Essai d'une critique de la vie et d'une science de la pratique. Paris.

Arrieta Heras, B. (1993). Filosofía y ética en Maurice Blondel. Bilbao: Universidad de Deusto.

Baravalle, G. (1972). La pedagogia nel suo sviluppo storico. Roma: Paoline

Crippa, R. (1989). "Blondel, Maurice", en: Enciclopedia Pedagogica, Tomo I, pp. 1838-1845. Brescia.

D'Agostino, S. (1999). Dall'atto all'azione. Blondel e Aristotele nel progetto de "L'Action" (1893). Roma: PUG.

Grillo, A. (1970). "Blondel e la sua scuola", en Volpicelli, L., La pedagogia. Storia e problemi; maestri e metodi; sociologia e psicologia dell'educazione e dell'insegnamento. Milano.
Hourton, J. (1971). "Blondel y Blondelismo", en Gran Enciclopedia Rialp, Tomo IV, pp. 326-329. Madrid.

Hourton, J. (1961). "La filosofía de la acción de Maurice Blondel". Finis Terrae 8, pp. 54-69.

Isasi, J. M. (1996). "Maurice Blondel, el filósofo de la acción", en Blondel, M. (1996), La Acción. Ensayo de una crítica de la vida y de una ciencia de la práctica, pp. XIII-XLIV. Madrid: BAC.

Leclerc, M. (2000). Il destino umano nella luce di Blondel. Assisi.

Marion, J. L. (1990). "La conversion de la volonté selon L'Action". En : Folscheid, D. (ed.)(1989) Maurice Blondel. Une dramatique de la modernité. pp. 154-165. Paris.

Reale, G. ; Antiseri, D. E Laeng, M. (1995). Filosofia e pedagogia dalle origini ad oggi, Tomo III. Brescia. 
